\title{
Methodology for computer-assisted optimization of waste flow
}

\author{
Cicerone Laurentiu Popa $^{1,{ }^{*}}$, Costel Emil Cotet $^{1}$, Radu Constantin Parpala ${ }^{1}$, and Tiberiu \\ Dobrescu ${ }^{1}$ \\ ${ }^{1}$ University Politehnica of Bucharest, MSP Department, Spl. Independentei 313, Bucharest, Romania
}

\begin{abstract}
The paper reports the development of a methodology based on computer simulations with the purpose to support decisions in designing the optimal architecture of different types of selective waste collection systems and recycling systems. The design of such systems is a complex task which involves both a very good knowledge of selective waste collection system equipment characteristics and of recycling processes, and the correct placing of the equipment along the flow so that to avoid underutilization of the structural elements and to avoid bottlenecks which generate low productivity or even blockages. The methodology is applied for three case studies in which different types of waste flow models are investigated: hybrid waste flows (windshields recycling), discrete waste flows (waste electric and electronic equipment collection) and continuous flows (industrial and automotive used oil collection and recycling). The architectures of these systems are optimized using the developed methodology in order to increase usage degree and productivity.
\end{abstract}

\section{Introduction}

In a very comprehensive view, the methodology proposed in this paper comprises optimization techniques for procurement, usage, handling, transformation and disposal of physical mobile entities like different types of waste in waste collection systems and recycling systems. The area of this methodology application is wide and implies concepts of the material flow theory [1]. An analysis of the literature in the field of MFT showed that the general focus is on presenting applications/case studies in different areas and, thus, obtaining specific conclusions, while the theoretical approaches and paradigms of MTF are considered only in few studies [2-4].

The material flow theory (MFT) proposes a general scientific model without taking into account the nature of the described material flow. Even if some of the considerations made are covering the entire MFT area, the main focus of the paper does not concern the specific aspects of economic or social material flow, but only on the waste flow models area of applications. Therefore, in the present paper MFT will be used in a restricted sense, as a set of simulation and optimization rules for productivity duration and costs using various virtual waste flow models.

\footnotetext{
${ }^{*}$ Corresponding author: laur.popa79@gmail.com
} 


\section{Optimizing different types of waste flow models. A synoptic view}

The proposed algorithm for optimizing recycling architectures performances should be adapted to the specific requirements of waste categories by specific types of selective waste collection systems and recycling systems. A waste processing architecture (WPA) can be defined as a set of structural elements interacting such that to contribute by specific processes to waste recycling. There are four main structural elements (work points, transport systems, transfer systems, buffers) and two auxiliary structural elements (mobile entities and human resources) in a waste processing architecture. Different types of waste flow models are considered: hybrid, discrete and continuous waste flow. For each one of these categories, establishing a WPA for a specific or a family of waste models involves designing and structuring the relationships between structural elements, processes, procedures and human resources. That is both defining these elements, placed and acting in a specific layout, as well as the material and information flow between them.

We agree to define here the role of the structural elements as follows. The work points are the structural elements covering all the technological stages necessary for the waste processing. Transport systems are moving the mobile entities between the long distance situated structural elements. The transfer systems are orienting and fixing the mobile entities, making as well the connection between near structural elements. Buffers are storing the mobile entities for a limited period of time. The mobile entities constitute the waste flow. The human resources are involved in two postures: operational (acting like a worker) and monitor (supervising the activity of the WPA).

The advances in three-dimensional computer-aided design (3D CAD software) made possible the creation and use of databases containing parameterized virtual models of all the structural elements that can be included in a WPA. These databases will be made available by producers (being already included in software applications) and their items can be put together in order to form a virtual model of a WPA. This virtual model should precisely reproduce the real system structural elements, in terms of overall dimensions and reciprocal positions and placement in the layout, being essential in optimizing the WPA. The modifications in the WPA needed after different simulation runs are easy to perform due to the parameterization of the virtual models, therefore decreasing the total optimization time.

The modelling and simulation algorithms for optimizing WPA using MFT are different according with two main categories as follows. A diffused WPA can be defined as systems with more than two work points connected by transport \& transfer systems and using deposits at local or system level. A concentrate WPA can be defined as systems based on a single work point surrounded \& assisted by transport, transfer \& deposit facilities.

In a work point, one or several operations/activities can take place using processes and machines. In an attempt to decrease the overall production time, there are approaches which consider eliminating the transport \& transfer times between work posts by combining in a single machine, two (or more) processes [1].

The three main processes categories are: subtractive - involving material removal, additive - involving material addition layer-by-layer and with material distribution. Additive and subtractive processes can be combined [1] within a single machine, completely computer-controlled, in order to benefit of the advantages of each technology, such as complex geometry, controlled geometry, functional graded material parts - for additive processes, and high precision and surface quality or very good mechanical properties - for subtractive processes.

From the point of view of the MFT, these hybrid processing systems, currently gaining more interest and importance, are considered as concentrated architectures, and can be modelled and optimized accordingly. As examples of hybrid processing can be mentioned: 
solid recovered fuel systems [5, 6] or glass recycling systems [7]. The prosed algorithm to optimize WPA by increasing usage degree and productivity customizes general algorithms used in MFT. The main steps are starting from this point and are based on all the above information, as follows.

A preliminary processing architecture virtual modelling is done with the necessary parameters for the work points, buffers, transport and transfer systems. The trajectories of the mobile entities (parts and tools) are established. For this preliminary architecture, virtual prototype the material flow is simulating for a performance diagnosis report [8]. Using this report, the flow concentrators could be identified. There are two kinds of solutions for eliminating these concentrators by modifying the WPA model [9].

Functional remodelling potentiates the WPA performance by modifying the structural elements layout or functional parameters but is not introducing or eliminating structural elements.

Technological remodelling allows WPA changes also when structural elements should be added or removed. After functional or technological remodelling, another material flow simulation is performed for quantifying the increasing performance of the optimized WPA. The last stage is the profit validation based on the comparison of the investment in optimizing the WPA and the gain from the increased system performance.

\section{Case studies}

The methodology is applied for three case studies in which different types of waste flow models are investigated: waste electric and electronic equipment collection as an example for discrete waste flows, industrial and automotive used oil collection and recycling as an example for continuous flows, windshields recycling as an example for hybrid waste flows. First and second case studies are part of the same research project with the subject of selective waste collection. The third one refers to a research project in the field of waste processing.

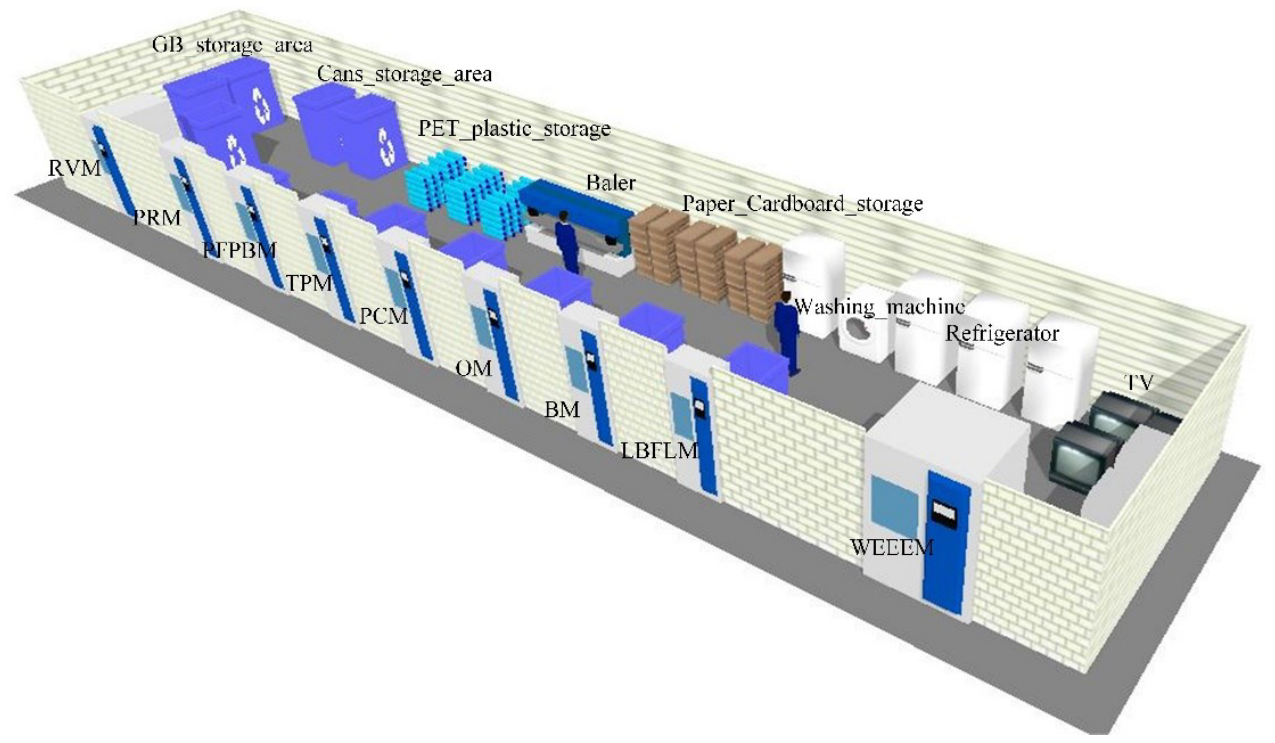

Fig. 1. The 3D model of the current selective waste collection system made in Witness Horizon 
The three-dimensional virtual model of the selective waste collection system made in Horizon Witness is presented in figure 1, WEEEM (waste electric and electronic equipment module) being a part of it. The systems include also:

- RVM module (Reverse Vending Machine) for receiving, identifying and sorting the glass recipients, plastic recipients and aluminium cans;

- PRM (plastic recipients module) for plastic recipients over 5 litres;

- PFPBM - Plastic film and plastic bags module;

- TPM - Tetra Pack module;

- PCM - Paper and Cardboard module;

- OM - Oil module;

- BM - Battery module;

- LBFLM - Light bulb and fluorescent lamp module.

These modules are not automated. Two operators are receiving the collected waste. The operators are used for loading, weighing, voucher issuance and storage of WEEEs (including refrigerators, washing machines, TVs, phones, laptops, PC drives, printers, video consoles, microwave etc.).

The three-dimensional virtual model is used to perform a waste flow simulation in order to conduct a diagnosis. The waste flows from the system are optimized using the developed methodology to increase usage degree and productivity. For the WEEEM a technological remodelling was made to obtain an automated module. The module for collecting electrical equipment and electronic waste was divided in two separate modules:

- one for big electronics and home appliances. This will take over the refrigerators, washing machines, TV sets and after identification and weighing they will be transported by a conveyer to the transfer zone, from where they will be picked up by the AS/RS (Automated storage and retrieval system) and deposited in the storage area;

- one for small electronics and home appliances. This will take over the phones, laptops, PC drives, printers, video consoles, microwave and after identification and weighing they will be transported by a conveyor to the transfer zone, point from which they will be picked up by the AS/RS system and will be deposited in the storage area.

The data obtained after the optimization shows an increase in terms of usage degree and productivity

The oil module is included in the current waste collecting system, but is available only in a few locations where industrial and automotive used oil collection can be done. Now, the oil is collected in two different tanks (one for industrial an automotive oil and one for cooking oil). The operators are receiving the containers with oil from the customers and put it in tanks. After the preliminary simulation, the flow was have modified by including a RVM for oil in the system. Also, the oil recycling system was added in simulation as an example for continuous flow simulation.

The recycling process for cooking oil start with the identification of free fatty acids (FFA) percent, if FFA is larger than $2 \%$ then the FFA must be reduced by using an acidcatalysed esterification process. Using a trans esterification process based on sodium hydroxide and methanol reduces the oil viscosity. After trans esterification, the methanol is removed in a washing process. Once separated the biodiesel is purified. At the end the biodiesel is dried to remove all water remaining from the washing process [10]. The flow is shown in figure 2 .

The results show possible usage increase degree of the system due to the fact of introducing the RVM machine. In this case the waste collection can be done 24 hours/7 days every week and is not limited to the presence of operators. 


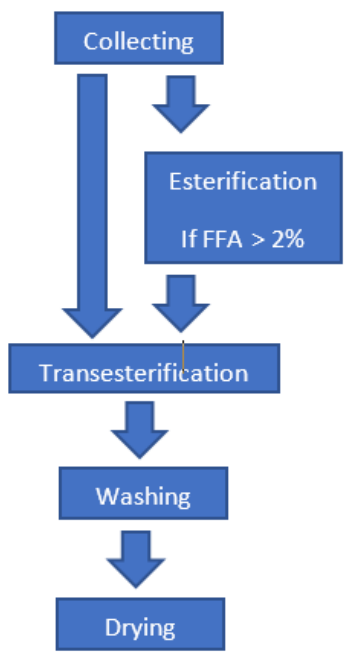

Fig. 2. Cooking oil recycling flow

The third case study refers to windshields recycling as an example for hybrid waste flows. The windshield glass material flow and the recycling installation virtual model made in Witness Horizon are shown in figure 3.

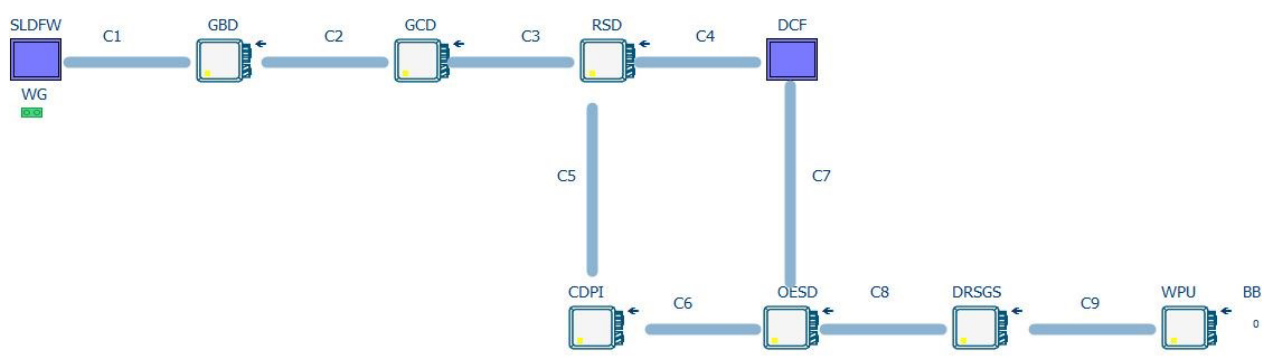

Fig. 3. The 2D virtual model of the windshield glass recycling installation made in Witness Horizon

WG (windshield glass) is introduced in the recycling system using the supply/loading device with glass waste (SLDFW). C1 conveyor will transport the WG to the glassbreaking device (GBD). From the Glass crusher device (GCD), the glass waste is transported to the rotating sieve device (RSD) using the $\mathrm{C} 3$ conveyer. Glass waste is transported to CDPI (Crushing device of pieces with impurities) by the C5 conveyer and PVB (laminated glass with polyvinyl butyral) waste is transported to DCF by the C4 conveyer. OESD (Optical-electrical sorting device) will sort the glass with impurities and using the $\mathrm{C} 7$ conveyer the waste will be transported to DCF (Catching device of fractions resulted by the recycling process). Clean cullets are sorted on DRSGS (device with rotating sieve for the glass sorting) depending on the desired granulation. On the Weighting and packaging unit (WPU) the granules are stored in big bags with $1000 \mathrm{~kg}$ capacity.

The preliminary simulation results show bottlenecks on GCD (22\%) and RSD (17\%). The functional remodelling was used to remove the bottlenecks and to increase the system productivity. The reports after system's optimization show that 3 big bags (BB) were obtained and at WPU during the packaging and another big bag are at $69 \%$ of capacity (approx. $690 \mathrm{~kg}$ ). The quantity that the installation will process during one hour is 3.69 tonnes (an average of 26 tonnes per day -7 hours). 


\section{Conclusions}

Current needs and practice in industrial engineering determined us to adapt the general MFT and to develop specific algorithms for diagnosis and optimization for WPA.

As can be noticed, our approach accommodates the advances in digital fabrication and the development of new manufacturing processes/technologies to waste processing.

When designing the layout of WPA, virtual models of different structural elements can be used, while in simulating the material flow, data from processes simulations can constitute input parameters. In this context of using data from different types of applications for defining and optimizing WPA, data compatibility and data portability issues should not be neglected when choosing the software tools for supporting the optimal definition of a WPA.

The core of the proposed methodology assumes than in dealing with MFT applied to WPA, one should consider using a specific algorithm for optimizing the associated architecture by modelling and then simulating the material flow trajectory within the system. Such an algorithm, as detailed in the previous section, offers a structured modality to increase the productivity and profit in WPA in order to respond to different requirements.

Based on the results described above in this paper the authors established the main future research direction as improving performances analysis by developing new measuring tools and integrating the investment analysis in the material flow simulators.

This work was supported by a grant of the Romanian National Authority for Scientific Research and Innovation, CNCS/CCCDI - UEFISCDI, project number PN-III-P2-2.1-BG-2016-0437, within PNCDI III.

\section{References}

1. C. E. Cotet, D. Popescu, Enc. Inf. Sci. Tech., 3786 - 3794 (2014)

2. J. V. Steinaecker, G. Jürgens, Env. Inf. Sys. Ind. Pub. Adm., 356-366 (2001)

3. G. A. Swanson, Ent Inf. Sys., 2, 21-31 (2008)

4. S. Xu, Inf. Sys. Fr., 10, 601-609 (2008)

5. W. L Kepplinger, T. Tappeiner, Was. Man Re., 30, 450-453 (2012)

6. K. E. Lorber, R. Sarc, A. Alexia, Was. Man Re., 30, 370-383 (2012)

7. J. R Mueller, M. W. Boehm, C. Drummond, Was. Man., 32, 1560-1565 (2012)

8. C. L. Popa, C. E. Cotet, V. Ionita, St. Gavrila, Proc. Eng., 100, $334-339$ (2015)

9. C. E. Coteț, C. L. Popa, G. Enciu, A. Popescu, T. Dobrescu, Int. J.Simul. Model, 15, 5-16 (2016)

10. N. H. Abdullah, S. H. Hasan, N. R. M. Yusoff, Int. J. Mat. Sci. Eng., 1, $94-99$ (2013) 